\title{
Quantum dot photonic crystal detectors
}

Kalyan Teja Posani, Vaibhav Tripathi, Senthil Annamalai, Sanjay Krishna, Raviv Perahia, et al.

Kalyan Teja Posani, Vaibhav Tripathi, Senthil Annamalai, Sanjay Krishna, Raviv Perahia, Orion Crisafulli, Oskar Painter, "Quantum dot photonic crystal detectors," Proc. SPIE 6129, Quantum Dots, Particles, and Nanoclusters III, 612906 (7 February 2006); doi: 10.1117/12.641750

Event: Integrated Optoelectronic Devices 2006, 2006, San Jose, California, United States 


\title{
Quantum Dot Photonic Crystal Detectors
}

\author{
Kalyan Teja Posani, Vaibhav Tripathi, Senthil Annamalai and Sanjay Krishna \\ Electrical and Computer Engineering Department, Center for High Technology Materials, \\ University of New Mexico, Albuquerque, \\ New Mexico 87106 \\ Raviv Perahia, Orion Crisafulli and Oskar Painter \\ Caltech applied Physics, California Institute of Technology, 1200 East California Boulevard, \\ Pasadena, California 91125
}

\begin{abstract}
In this paper we report the use of a photonic crystal resonant cavity to increase the quantum efficiency, detectivity $\left(\mathrm{D}^{*}\right)$ and the background limited infrared photodetector (BLIP) temperature of a quantum dot detector. The photonic crystal is incorporated in InAs/InGaAs/GaAs dots-in-well (DWELL) detector using Electron beam lithography. From calibrated blackbody measurements, the conversion efficiency of the detector with the photonic crystal (DWELL-PC) is found to be $58.5 \%$ at $-2.5 \mathrm{~V}$ while the control DWELL detectors have quantum efficiency of $7.6 \%$ at the same bias. We observed no significant reduction in the dark current of the photonic crystal devices compared to the normal structure. The generation-recombination limited $\mathrm{D}^{*}$ at $77 \mathrm{~K}$ with a $300 \mathrm{~K} \mathrm{~F} 1.7$ background, is estimated to be $6 \times 10^{10} \mathrm{cmHz}^{1 / 2} / \mathrm{W}$ at $-3 \mathrm{~V}$ bias for the DWELL-PC which is a factor of 20 higher than that of the control sample. We also observed a $20 \%$ increase in the BLIP temperature for the DWELL-PCs.
\end{abstract}

Keywords: Photonic crystal, Resonant Cavity, Quantum dots, Infrared sensors, Detectors, Focal plane arrays, Conversion Efficiency, Detectivity, Dark current.

\section{INTRODUCTION}

Quantum dot infrared photo detectors (QDIP) have been explored extensively in the past few years due to their potential to provide low dark current ${ }^{1-6}$, normal incidence operation ${ }^{1,2}$ and high operating temperatures ${ }^{6,7}$. The three dimensional confinement of electrons in a quantum dot reduces the thermionic emission ${ }^{4,8}$, thus resulting in low dark current and the high energy relaxation time ${ }^{3}$ due to the phonon bottleneck enables high temperature operation. Recently long wave infrared focal plane arrays based on self-assembled QDs have also been fabricated ${ }^{9}$. However, QD detectors suffer from low responsivity leading to lower quantum efficiency as compared to Quantum well infrared detectors. Also the tuning of operating wavelength in a QDIP requires a change in the growth process with different specifications, which is not commercially viable. To compensate for this disadvantage of different self assembly growth for different detectors, Dots-in-well (DWELL) structure has been proposed ${ }^{1,4,8,9,10,11}$. This DWELL structure has facilitated the tuning of operating wavelength by changing the quantum well thickness and reduced the dark current further. Efforts have been made to increase the quantum efficiency by using Diffracted Bragg's Reflector (DBR) in the DWELL structure. This 
creates a resonant cavity leading to photon entrapment in the active region resulting in good quantum efficiency. However this results in a very thick structure making it impractical for commercial applications. In this paper we report the use of a photonic crystal resonant cavity in the DWELL region to increase the responsivity while maintaining all the other merits of the QDIPs.

The idea behind the photonic crystal is the formation of a photonic bandgap by a spatial three-dimensional periodic variation of the refractive index ${ }^{12-16}$. The photonic crystal is realized by a hexagonal pattern of air holes in the active region as shown in figure2. The lattice parameter, which is the spacing between the air holes, is varied from $2.2 \mu \mathrm{m}$ to $2.4 \mu \mathrm{m}$ in steps of $0.05 \mu \mathrm{m}$. The three dimensional confinement of light, is attained by introducing localized defects by changing the radius of the air holes, perturbing the periodicity locally. The defect mode in a photonic crystal will serve as an effective resonant cavity, since it would trap light in a very narrow frequency band and would hardly suffer any losses ${ }^{12,15,16}$. In this paper we discuss the modeling of the photonic crystal pattern followed by the characterization. A comparison of performance between the photonic crystal detector and a normal DWELL detector with the same structure is presented.

\section{EXPERIMENT}

InAs/InGaAs/GaAs dots-in-well (DWELL) detectors were grown by molecular beam epitaxy on semiinsulating GaAs substrates. The active region consists of 15 stacks of 2.4 monolayers (ML) of n-doped InAs quantum dots placed in an $\operatorname{In}_{0.15} \mathrm{Ga}_{0.85} \mathrm{As} / \mathrm{GaAs}$ quantum well ${ }^{1,9}$ as shown in the figure1. The dots were doped $\mathrm{n}$-type with a silicon concentration of $3 \times 10^{10} / \mathrm{cm}^{2}$ at a growth rate of $0.053 \mathrm{ML} / \mathrm{s}$, which is equivalent to one electron per dot. The GaAs layers on either side of the active region, grown at $580^{\circ} \mathrm{C}$, have a doping concentration of $2 \times 10^{18} / \mathrm{cm}^{3}$ and they serve as contact layers. The structure was then processed into $400 \mu \mathrm{m}$ square mesas with active region apertures ranging from $25 \mu \mathrm{m}$ to $300 \mu \mathrm{m}$ in a class-100 clean room using photolithography, metallization, etching and annealing techniques. The top and bottom contacts were annealed at $400^{\circ} \mathrm{C}$ with $\mathrm{Ge} / \mathrm{Au} / \mathrm{Ni} / \mathrm{Au}$ alloy as the contact metal.

The band structure of the photonic crystal cavity was modeled using plane wave expansion methods and the effective index of the fundamental TE and TM modes of the unpatterned quantum dot heterostructure found using Finite-difference techniques. From the band structure analysis certain high symmetry points in the Brillouin zone are targeted to lower the conduction band mode frequency or to raise the valence band edge mode frequency using the two geometrical parameters lattice spacing ' $a$ ' and the hole radius ' $r$ '. Localized defects are introduced in the photonic crystal pattern by perturbing the radius of the air holes at some locations in the waveguide and the defect mode frequency is estimated to be around 0.3 from finite difference time domain simulations. This confines the lattice parameter ' $\mathrm{a}$ ' to be around $2.4 \mu \mathrm{m}$ for a wavelength of $8.1 \mu \mathrm{m}$.

The photonic crystal cavity, with a hexagonal pattern of air holes, was defined using electron-beam lithography. The layout of the finished devices is shown in figure 2 and figure 3 shows the close-up view of the air holes in the active region. The wafer was diced and measurements were carried out on single pixel devices.

\section{RESULTS}

Spectral response measurements were done at $30 \mathrm{~K}$ for the photonic crystal detectors and the control sample using Nicolet FTIR spectrometer. The spectral plots at a voltage bias of $-3 \mathrm{~V}$ are shown in figure4. It can be observed from the data that the peak at $5 \mu \mathrm{m}$ is suppressed in the photonic crystal detectors as compared to the control sample. The photonic crystal sensors showed a strong peak at around $8 \mu \mathrm{m}$ and suppressed the other prominent peaks. This is evident from the fact that the photonic crystal resonant cavity traps light in a very narrow frequency band around the localized defect frequency, which leads to more absorption at that particular wavelength resulting in a dominant peak at the corresponding position in the spectrum. This result was corroborated from the laser experiment results where in a tunable laser source radiation instead of a blackbody is excited on the detectors to measure the spectral response.

The responsivity of these sensors, which is the amount of current produced per unit watt of incident power, is measured at $77 \mathrm{~K}$ by means of calibrated radiometry measurements using an $800 \mathrm{~K}$ blackbody and optical chopper setup and the spectral response data. The conversion efficiency of the detector which is the product of the quantum efficiency 
and the photoconductive gain is calculated from the responsivity and the peak wavelength by means of the following expression where $\mathrm{R}$ is the responsivity, $\eta$ the conversion efficiency and $\lambda$ being the peak wavelength.

$$
\eta=(\mathrm{Rhc}) /(\mathrm{q} \lambda)
$$

Figure5 shows the conversion efficiency plot for the photonic crystal sensors and the control sample. The conversion efficiency of the photonic crystal detectors is increased by about a factor of 10 as compared to the normal detector due to the influence of the resonant cavity.

Photo current and dark current measurements were made at different temperatures ranging from $30 \mathrm{~K}$ to $300 \mathrm{~K}$ in a bias range of $-3 \mathrm{~V}$ to $3 \mathrm{~V}$ using an $\mathrm{I}-\mathrm{V}$ curve analyzer. We observed the dark current increases with increase in temperature as expected due to more thermionic emission. The dark and photo current densities at $90 \mathrm{~K}$ are shown in the figure 6 for both the devices. The dark current in the photonic crystal devices was found to be lesser than the normal DWELL detector. This could be interpreted as due to loss of material in the active region, which was removed to make the hexagonal pattern of air holes. The Background limited infrared photodetector (BLIP) temperature of the detectors is calculated by finding the temperature at which the photocurrent equals the dark current. From the plots the BLIP temperature of the photonic crystal devices is obtained to be around $110 \mathrm{~K}$, an increase by about $30 \mathrm{~K}$ compared to the control detector, which shows that the photonic crystal detectors can work at higher temperatures than the normal DWELL detectors. Figure8 shows the Generation recombination noise limited detectivity $\left(\mathrm{D}^{*}\right)$ for both the photonic crystal and the normal DWELL detectors. The detectivity which is a measure of signal to noise ratio was found to be around 20 times higher for the photonic crystals sensors than the normal sample. A D ${ }^{*}$ of $6 \times 10^{10} \mathrm{cmHz}^{1 / 2} / \mathrm{W}$ at $-3 \mathrm{~V}$ bias has been reported for the photonic crystal detector when facing a 300K F1.7 background.

\section{CONCLUSIONS AND FUTURE WORK}

The use of photonic crystal structure in a DWELL detector increased its conversion efficiency by approximately 10 times. We comment only on conversion efficiency because of the non-unity photoconductive gain for the sample. Latest experiments predict that the gain lies in between 3-5. The increase in conversion efficiency takes care of the low quantum efficiency of DWELL detectors. The creation of photonic crystal cavity in a DWELL detector is easily done by electron beam lithography. This makes them easy to grow as compared to the Diffracted Bragg's Reflector making them more suitable for commercial applications. The increase in BLIP temperature clearly indicates that these devices can function at higher temperatures compared to the DWELL detectors. Increase in detectivity also points out an increase in signal with low dark current (noise). Therefore use of photonic crystals has not affected the advantage of low dark current in DWELL detectors. With these merits the Photonic Crystal Quantum Dot Infrared Photo Detector is an exciting prospect for future research.

The experimental results varied over samples with different aperture and radius to lattice parameter(r/a) ratio. The $50 \mu \mathrm{m}$ devices showed better performance as compared to the other $150 \mu \mathrm{m}$ photonic crystal devices. Thus optimization of the samples might be the next step in research. The experimental results mentioned in this paper might prove to be the start based on which further research may continue which makes these devices commercially viable.

The authors would like to acknowledge support from NSF ECS Grants 0428756/ 0401154/0434102. 


\section{FIGURES}

\begin{tabular}{|c|}
\hline $\operatorname{GaAs}\left(\mathrm{n}=2 \times 10^{18} \mathrm{~cm}^{-3}\right) 0.2 \mathrm{um}$ \\
\hline GaAs $500 \AA$ \\
\hline $\operatorname{In}_{15} \mathrm{Ga}_{85} \mathrm{As} 60 \AA$ \\
\hline InAs ODs $\left(\mathrm{n}=3 \times 10^{10} \mathrm{~cm}^{-2}\right) 2.4$ MLs \\
\hline $\operatorname{In}_{15} \mathbf{G a}_{85} \mathrm{As} 50 \AA$ \\
\hline GaAs $500 \AA$ \\
\hline $\operatorname{GaAs}\left(\mathrm{n}=2 \times 10^{18} \mathrm{~cm}^{-3}\right) 0.5 \mathrm{um}$ \\
\hline AlAs $300 \AA$ \\
\hline GaAs $2000 \AA$ \\
\hline GaAs S.I. Substrate \\
\hline
\end{tabular}

Fig. 1. Schematic of the DWELL detector, in which the photonic crystal cavity was inscribed

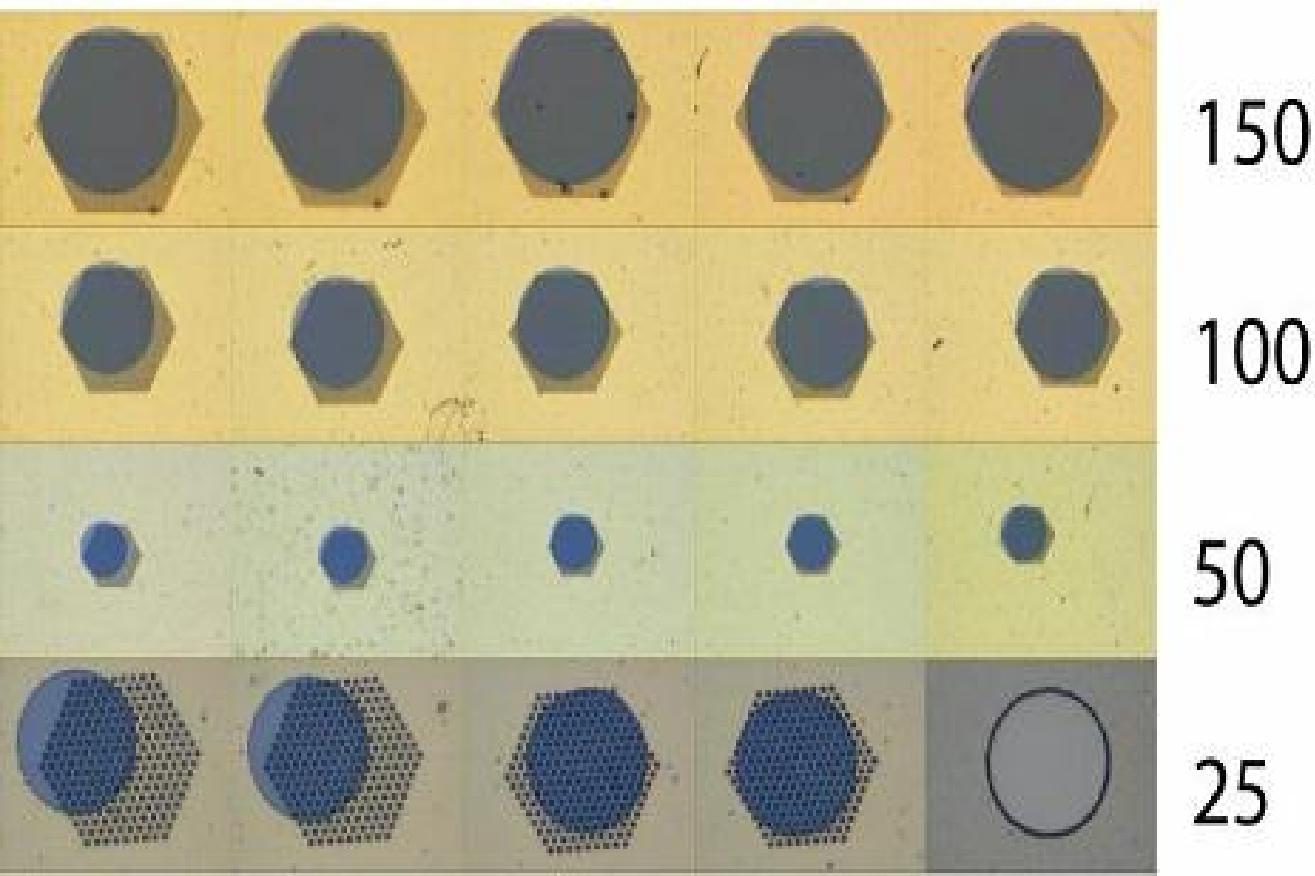

Fig.2 Layout of finished Devices 
$50 \mathrm{~m}$ Device

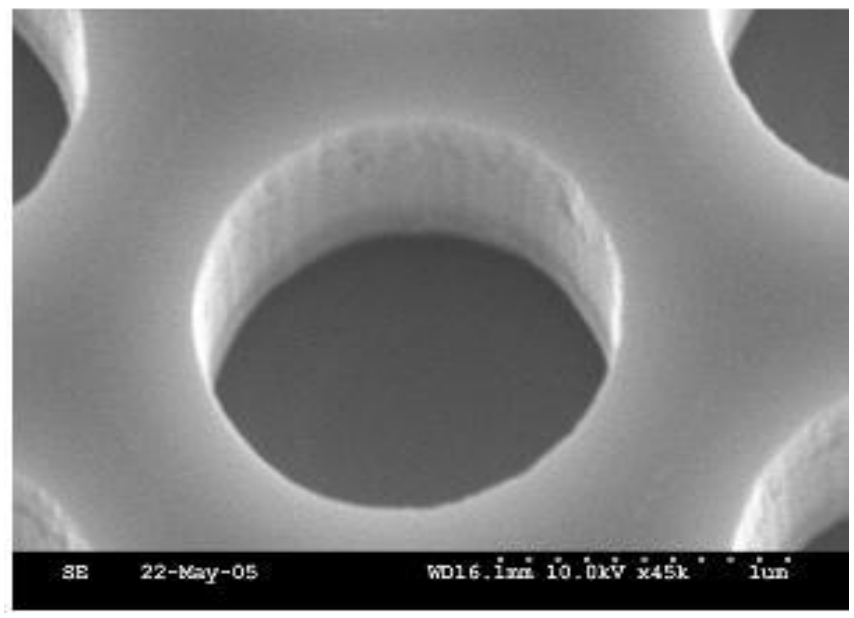

150um device



FIG. 3. Photonic Crystal Cavity 50um device (left), 150um device (right)

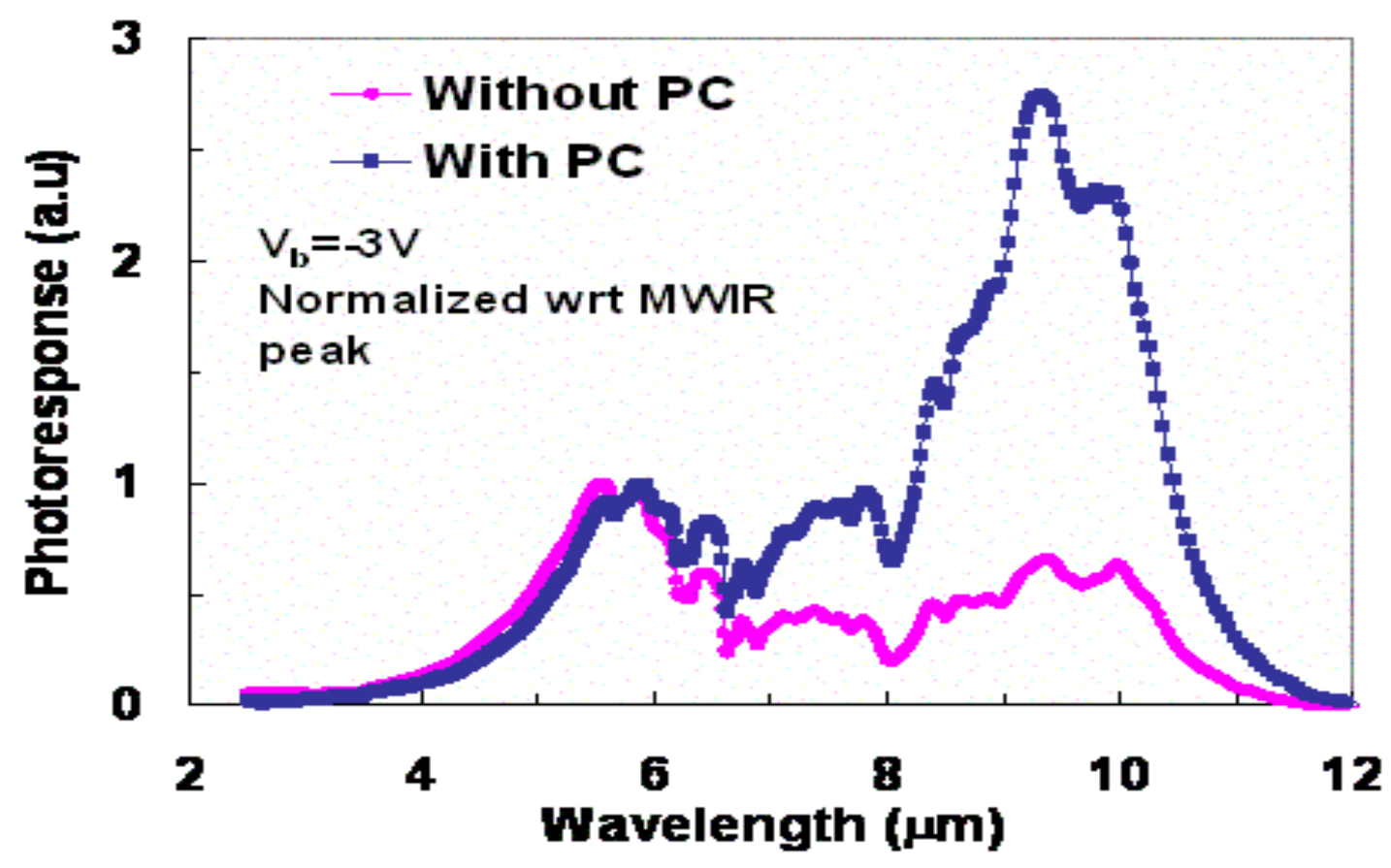

Fig.4. Spectral Response of the normal and photonic crystal devices at $-3 \mathrm{~V}$ 




\section{Bias(V)}

Fig.5. Comparision of the Conversion Efficiencies of the samples

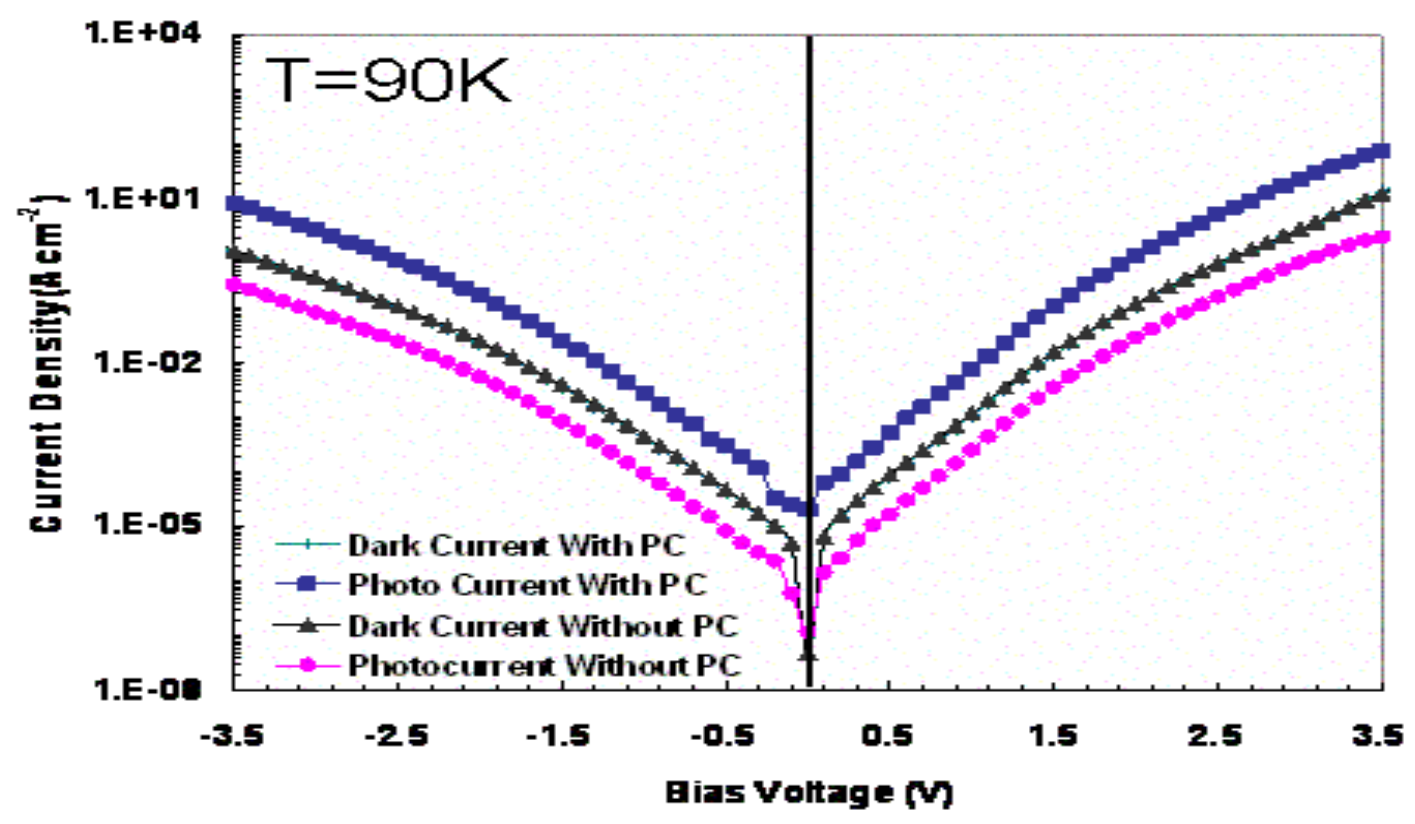

Fig.6. Dark and Photo current densities of the Control Sample and the Photonic crystal sensor 




FIG. 7. Comparision of the Detectivity $\left(\mathrm{D}^{*}\right)$ of normal and photonic crystal detectors

\section{REFERENCES}

1. S. Raghavan, D. Forman, P. Hill, N. R. Weisse-Bernstein, G. von Winckel, P.Rotella, S.Krishna, S. W. Kennerly and J. W. Little, "Normal-incidence $\mathrm{InAs} / \mathrm{In}_{0.15} \mathrm{Ga}_{0.85}$ As quantum dots-in-a-well detector operating in the long-wave infrared atmospheric window (8-12 $\mu \mathrm{m})$ ", J. Appl. Phys. 96(2),1036 (2004).

2. Zhengmao Ye, Joe C. Campbell, Zhonghui Chen, Eui-Tae Kim, and Anupam Madhukar, "Normal-incidence InAs self-assembled quantum-dot infrared photodetectors with a high detectivity", IEEE Quant. Elec. 38(9), 1234 (2002). 
3. B Kochman, A D. Stiff-Roberts, S Chakrabarti, J D. Phillips, S Krishna, J Singh, and P Bhattacharya, "Absorption, carrier lifetime, and gain in InAs-GaAs quantum-dot infrared photodetectors", IEEE Quant. Elec. 39(3), 459 (2003).

4. S. Krishna, S. Raghavan, G. von Winckel, A. Stintz, G. Ariyawansa, S. G.Matsik, and A. G. U. Perera, "Threecolor (lambda(p1)similar to $3.8 \mathrm{mu} \mathrm{m}$, lambda(p2)similar to $8.5 \mathrm{mu} \mathrm{m}$, and lambda(p3)similar to $23.2 \mathrm{mu} \mathrm{m}$ ) InAs/InGaAs quantum-dots-in-a-well detector”, Appl. Phys. Lett. 83(14), 2745 (2003).

5. Eui-Tae Kim, A. Madhukar, Zhengmao Ye and Joe C. Campbell, "High detectivity InAs quantum dot infrared photodetectors", Appl. Phys. Lett. 84(17), 3277 (2004).

6. M. D. Kim, S. K. Noh, S. C. Hong, and T. W. Kim, "Formation and optical properties of InAs/GaAs quantum dots for applications as infrared photodetectors operating at room temperature”, Appl. Phys. Lett. 82 (4), 553 (2003).

7. J. Phillips, Pallab Bhattacharya, S. W. Kennerly, D. W. Beekman, and M.Dutta, "Self-assembled InAs-GaAs quantum-dot intersubband detectors", IEEE Quant. Elec. 35(6), 936 (1999).

8. A.Amtout, S. Raghavan, P. Rotella, G. von Winckel, A. Stintz, and S. Krishna, "Theoretical modeling and experimental characterization of InAs/InGaAs quantum dots in a well detector”, J. Appl. Phys. 96(7), 3782 (2004).

9. S. Krishna, D. Forman, S. Annamalai, P. Dowd, P. Varangis, T. Tumolillo, A. Gray, J. Zilko, K. Sun, M. Liu, J. Campbell, D. Carothers, "Demonstration of a 320 x 256 Two-Color Focal Plane Array Using InAs/InGaAs Quantum Dots in a Well Detectors”, Applied Physics Letters, 86, 193501, 2005.

10. S. Krishna, S. Raghavan, G. von Winckel, P.Rotella, A.Stintz, C.P. Morath, D.Le, S.W. Kennerly, "Two color InAs/InGaAs dots-in-a-well detector with background-limited performance at 91K”, Applied Physics Letters, 82(16), 2003.

11. S. Krishna, "Quantum dots-in-a-well infrared photodetectors", J. Phys. D: Appl. Phys. 38 (2005) 2142-2150

12. J. D. Joannopoulos, R. D. Meade, J. N. Winn, Photonic Crystals, Princeton University Press, Princeton, New Jersey, 1995.

13. O. Painter, R. K. Lee, A. Yariv, A. Scherer, J. D. O.Brien, P.D. Dapkus, I. Kim, "Two-dimensional Photonic Crystal Defect Laser", Science, 1819-1821, 284(5421), Jun. 1999.

14. P Yu, J Topolancik, P Bhattacharya, "Characteristics of a Photonic Crystal Defect Waveguide-Coupled Quantum-Dot Photodiode", IEEE Journal of Quantum Electronics, 2004

15. S.G. Johnson, J. D. Joannopoulos, Photonic Crystals, the road from theory to practice, Kluwer Academic publishers, Boston, 2002.

16. K. Inoue, K. Ohtaka, Photonic Crystals physics, Fabrication and Applications, Springer series, New York 2004. 